\title{
Flares and the chromosphere
}

\author{
Hugh S. Hudson ${ }^{1,2}$ and Lyndsay Fletcher ${ }^{2,1}$ \\ ${ }^{1}$ SSL, UC Berkeley, CA 94720-7450, USA \\ ${ }^{2}$ University of Glasgow, $U K$
}

(Received December 2, 2007; Revised August 24, 2008; Accepted August 31, 2008; Online published May 29, 2009)

\begin{abstract}
The chromosphere (the link between the photosphere and the corona) plays a crucial role in flare and CME development. In analogies between flares and magnetic substorms, it is normally identified with the ionosphere, but we argue that the correspondence is not exact. Much of the important physics of this interesting region remains to be explored. We discuss chromospheric flares in the context of recent observations of white-light flares and hard X-rays as observed by TRACE and RHESSI, respectively. We interpret key features of these observations as results of the stepwise changes a flare produces in the photospheric magnetic field.
\end{abstract}

Key words: Solar flares, solar chromosphere, solar corona, Alfvén waves.

\section{Introduction}

The chromosphere historically has been the origin of much of what we understand about solar flares. The reason for this was the recognition, in the 19th and early 20th centuries, of the extreme sensitivity of $\mathrm{H} \alpha$, a strong Fraunhofer absorption line formed in the chromosphere, to solar magnetic activity. Spectroscopic observations of this line and its imaging led to exciting discoveries regarding active prominences, ejecta, flare brightenings etc. (Hale, 1930). Eventually it was realized that the original flare observation of 1859 (Carrington, 1859) was simply the tip of the iceberg, and that the entire solar atmosphere was participating in events that have now come to be defined more by their coronal X-ray emission (the GOES classification) rather than their $\mathrm{H} \alpha$ importance levels (Thomas and Teske, 1971). Research attention, indeed, has largely left the chromosphere layers in favor of coronal and even interplanetary effects (CMEs and ICMEs; see Schwenn, 2007, for a recent review).

Our understanding of the chromosphere, until recently, has been limited to the "semi-empirical" models, based on 1D radiative-transfer physics. Such an approach omits dynamics except for the "microturbulence" factor and much of the interesting plasma physics; for example these models assume $T_{\mathrm{e}}=T_{\mathrm{i}}$ everywhere. See Berlicki (2007) and Hudson (2007) for recent reviews about the flaring chromosphere, and for references to the abundant literature on this subject.

In the often-discussed but imperfect analogy between solar flares and auroral substorms, the chromosphere plays the role of the ionosphere, but these regions have substantially different properties and the detailed physics may not produce analogous effects (e.g., Haerendel, 2007). On the larger scale there is also no analog of the solar wind flow-

Copyright (c) The Society of Geomagnetism and Earth, Planetary and Space Sciences (SGEPSS); The Seismological Society of Japan; The Volcanological Society of Japan; The Geodetic Society of Japan; The Japanese Society for Planetary Sciences; TERRAPUB ing around an active-region field concentration in the solar corona, so that the convective $(\mathbf{v} \times \mathbf{B})$ electric field across the geotail does not have an appropriate analog. The presence of a highly conductive solar atmosphere below the chromosphere also distinguishes it from the ionosphere. Thus, although striking observational parallels between flares and aurorae have been noted by many authors (e.g., Obayashi, 1975), the basic physics may be quite different in regard to causation or the dynamical development of the phenomena. In this paper we touch on flare energetics (Section 2), energy build-up (Section 3), and energy release (Section 4), attempting to use magnetospheric concepts as a guide to understanding.

\section{Chromospheric Flare Energetics}

The radiative energy of a solar flare appears mainly in the optical and UV continuum, which form in the lower solar atmosphere, most probably the chromosphere (e.g. Allred et al., 2005; Fletcher et al., 2007). This is in spite of the fact that the chromosphere itself (for this purpose, all regions of the solar atmosphere between photospheric and coronal temperatures) cannot contain sufficient energy to power a flare (see Hudson, 2007, for discussion). For example, the gravitational energy contained in coronal filaments does not play a strong role in flare energization. The radiated flare energy appears in compact emission patches that our current observations do not resolve either in space or in time (Hudson et al., 2006), and Fletcher et al. (2007) have confirmed that the immediate source of the radiated energy lies in the electrons accelerated in the impulsive phase of the flare. Of this energy the chromospheric $\mathrm{H} \alpha$ component and the coronal soft X-ray component each comprise less than about $10 \%$ of the total (Thomas and Teske, 1971).

Zeeman-splitting observations (Wang, 1993; Sudol and Harvey, 2005) have shown convincingly that flares result in large-scale perturbations of the photospheric magnetic field (see Fig. 1). This would generally be expected from any model of energy release from the coronal magnetic field, 


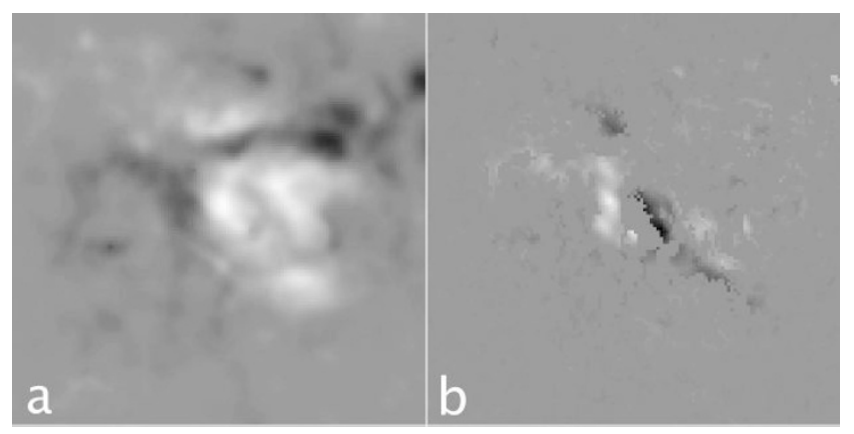

Fig. 1. GONG observations of the line-of-sight solar magnetic field prior to the X-class flare of 2003 October 29 (panel a); a difference map showing flare-related changes in the field (panel b); after Sudol and Harvey (2005). The field changes are of order 10\% of the line-of-sight field and can be detected in essentially all X-class flares, according to Sudol and Harvey.

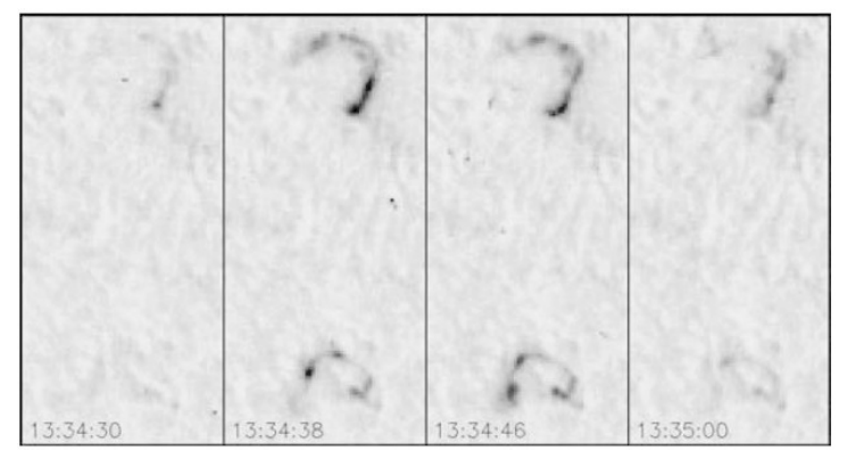

Fig. 2. Snapshots of TRACE observations (white light filter) of the C4.8 flare of 24 July 2004, showing the intermittency of the continuum emissions in both space and time. Spatial scale for each frame is $32^{\prime \prime} \times 68^{\prime \prime}$; the times shown on the figure span $30 \mathrm{~s}$ (from Hudson et al., 2006).

which will require restructuring in order to reduce the stored magnetic energy $\int\left(B^{2} / 8 \pi\right) d V$ (e.g., Hudson, 2000), as for example with large-scale magnetic reconnection. The main contribution to the coronal magnetic energy and its stress are concentrated strongly in the lower solar atmosphere (e.g., Régnier and Priest, 2007). Thus we need a theoretical understanding of how the coronal stored energy can flow to and focus itself into the chromospheric emission regions (see Fig. 2). Heretofore this coupling has been understood as the result of beams of electrons coming from an unknown coronal acceleration site, for which there are several possibilities (e.g., Miller et al., 1997). These ideas underlie the "thick target" model for the impulsive phase of a flare, which envisions electron beams capable of transporting energy from the coronal storage site into the dissipation regions (Brown, 1971; Hudson, 1972). We do not know yet how the particle acceleration relates to the magnetic restructuring needed to release coronal energy.

\section{Cross-field Currents}

Another aspect of flare energetics and the chromosphere is the mapping of subphotospheric magnetic twist into coronal currents (Longcope and Welsch, 2000). This in principle involves the use of the full conductivity tensor, though there is little discussion of this yet in the solar literature (e.g., Kazeminezhad and Goodman, 2006; Arber et al., 2007) at least as regards flares. Haerendel (2007) points out that the large ion-neutral coupling in the chromosphere makes the perpendicular conductivity is smaller than the parallel conductivity, at least for slowly-varying currents, and yet cross-field current systems must develop slowly in such a way as to match the conflicting boundary conditions at the two independent footpoints of a coronal flux tube. Auroral models make use of ionospheric currents to close coronal current systems, but in the case of the Sun we believe that the significant currents are injected through the photosphere in a slowly-evolving manner, and that these currents serve to energize the non-potential fields in the corona. In this sense the chromosphere must play the roles of both the ionosphere and the magnetopause.

We can write the perpendicular conductivity as

$$
\sigma_{\perp, x}=\frac{N e^{2}}{m_{\mathrm{e}}} \frac{v_{x n}}{v_{x n}^{2}+\omega_{c x}^{2}}
$$

with $x$ representing either ions or electrons (Banks, 1966), where $\omega_{c x}^{2}$ represents the Larmor frequency for particle species $x$ and $n$ denotes neutrals. The neutral collision frequencies $v_{x n}$ largely determine the perpendicular conductivity and it is not clear at present which particle species dominates the steady-state perpendicular current system. In any case for "normal" chromospheric and coronal conditions, as inferred from standard semi-empirical models, the Larmor frequencies greatly exceed the neutral collision frequencies so that the perpendicular conductivity is small relative to the parallel term. So far as we are aware, the questions posed by the requirement to establish slowly varying cross-field currents in the chromosphere have not been discussed in the literature, and we do not know the role that they play in energy storage or dissipation. 


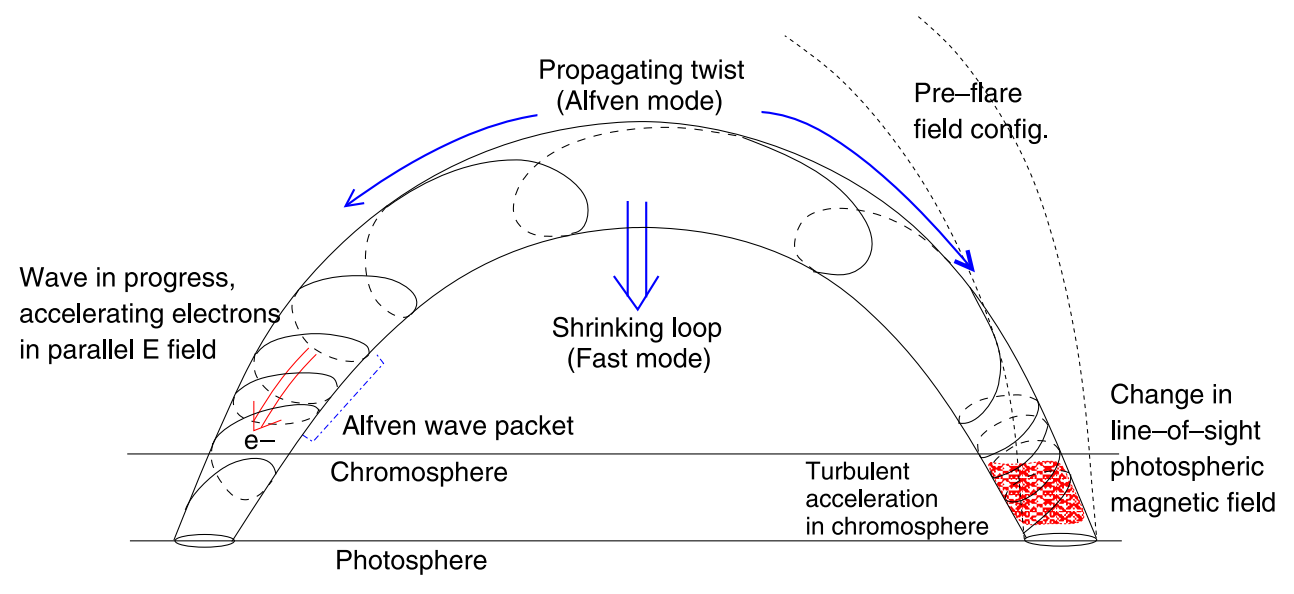

Fig. 3. Sketch of flare mechanisms as discussed in the text. Deformation of a flux tube results in Alfvén waves that transport energy into the footpoint regions via the Poynting flux. The wave deformation also perturbs the global structure to produce effects such as those seen in Fig. 1 . Particle acceleration results either directly from parallel electric fields produced by the waves, or in tubulent cascades developing from their interactions.

\section{Field Restructuring, Waves, and Energy Trans- port}

Changes of the coronal magnetic structure imply the transport of energy via Poynting fluxes (Melrose, 1992). The observations indicate that coronal energy dissipates in the chromosphere, and the hard X-ray signature directly implicates weakly relativistic electrons. Thus the magnetic restructuring, and the energy transport it implies, must somehow result in the acceleration of electrons to non-thermal energies. We sketch how this may happen in Fig. 3 (Fletcher and Hudson, 2008). The sudden reconfiguration of the field, in the ideal MHD approximation, would launch Alfvén waves. Emslie and Sturrock (1982) argue that the suddenness of flare energy release requires that the Alfvén mode and the fast mode predominate in the partition of this energy. The Alfvén mode is particularly interesting in this context, because as a transverse wave its Poynting flux $\mathbf{E} \times \mathbf{B}$ must be strictly parallel to $\mathbf{B}$ and thus be strongly ducted into the footpoint regions.

The mechanism for electron acceleration remains illunderstood. Because it is energetically so important (Kane and Donnelly, 1971; Lin and Hudson, 1971), its identification is fundamental to understanding the physics of solar flares. In the view of Fig. 3, the acceleration must happen as a result of the Alfvén-wave energy flux ducted along the arcade loops that result from the restructuring. This suggests several possible acceleration mechanisms, some of which have been recently reviewed by Miller et al. (1997).

Our scenario suggests additional acceleration mechanisms. Alfvén waves in the lower corona may propagate dispersively (e.g., Stasiewicz et al., 2001), inducing parallel electric fields directly. If the plasma beta, $\beta=$ $2 n k T /\left(B^{2} / 8 \pi\right)$, is smaller than $m_{\mathrm{e}} / m_{\mathrm{i}}$, where $m_{\mathrm{e}} / m_{\mathrm{i}}$ is the electron/ion mass ratio, then the Alfvén mode can become dispersive in the form of a kinetic Alfvén wave and develop a parallel field directly. Fletcher and Hudson (2008) review how this happens. The waves may also cascade into forms of turbulence suitable for stochastic particle acceleration, and this cascade may develop promptly under some conditions. First-order Fermi acceleration and the betatron effect may also play roles in the "collapsing trap" (e.g., Veronig

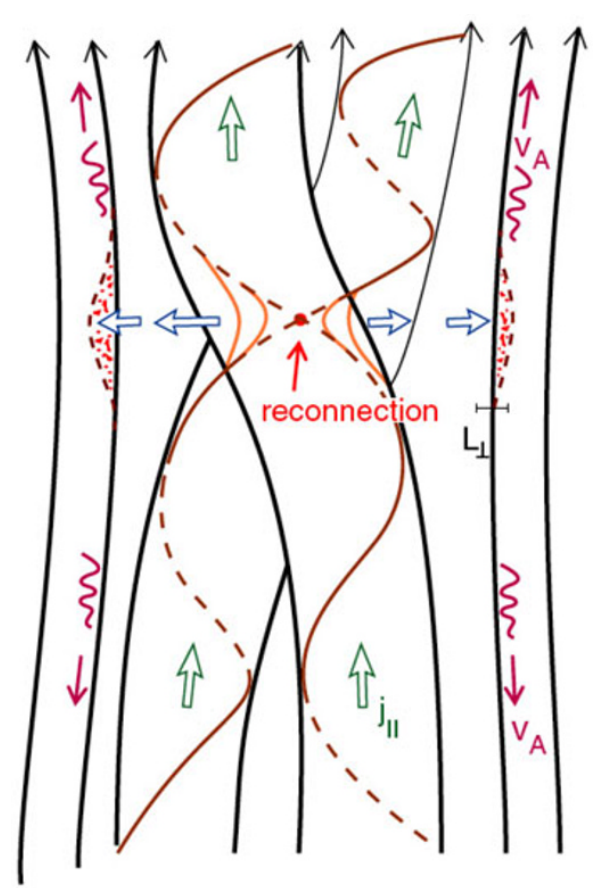

Fig. 4. Sketch showing how magnetic reconnection may excite Alfvén waves. From Haerendel (2007).

et al., 2006), and finally the disruption may create shock waves that may also accelerate particles.

Our preferred scenario (Fig. 3) has the virtue of linking the observed magnetic-field variations with the powerful energy release seen in the visible and UV continua. The sketch by Haerendel (2007), reproduced in Fig. 4, illustrates the generation of Alfvén waves from the reconnection process. Particle acceleration, in this picture, could readily occur in the lower solar atmosphere, where the ambient electrons are numerous enough to overcome the "number problem" and concerns about electron beam dynamics. One weakness may be the apparent time-of-flight signature noted by Aschwanden (2002), which-though somewhat ambiguous - provides the main observational evidence for the existence of the intense coronal electron beams the 
thick-target model requires. Type III radio bursts also require beams, but of significantly lesser intensity.

\section{Conclusions}

Research in solar flares and terrestrial aurorae has long been stimulated by the observational analogies one can draw between the phenomena (e.g., Obayashi, 1975). The analogous elements include ribbon-like optical emissions, electron acceleration to $\mathrm{keV}$ energies, and similar magnetic geometries. There are observational differences though, and theoretically there also are good reasons not to have a strict analogy. Nevertheless we feel it important to discuss the physics of chromospheric flares, both in the energy build-up and release stages, in ways that exploit some of ideas auroral physics offers to the understanding of solar problems. We interpret the observed photospheric magnetic field changes as the result of large-scale Alfvén waves created during coronal magnetic restructuring (Fletcher and Hudson, 2008). Particle acceleration, a key observable in solar flares because of hard X-ray and $\gamma$-ray emission, then becomes secondary to the transport of energy via the Poynting fluxes of the waves. There are different ways in which the necessary particle acceleration may happen, most directly if the waves become dispersive in nature, but the identification of the acceleration mechanism remains an open problem.

Consideration of wave transport of energy in solar flares and CMEs seems like a logical and necessary development for the advancement of theoretical ideas. Some large-scale manifestations of waves are readily observable, originally as type II bursts and Moreton waves (e.g., Uchida et al., 1973), but now also at higher resolution in the EUV as the EIT waves (Thompson et al., 1999). The EIT waves introduce new kinds of behavior not seen before and it has become clear that not all of the motions can be identified with the Moreton-wave phenomenon (Biesecker et al., 2002). Small-scale waves such as those that could be directly responsible for particle acceleration are difficult to observe remotely, but their presence may be just as fundamental to solar-flare physics as comparable structures are in auroral physics. We therefore urge theoretical work involving the ideas discussed here.

Acknowledgments. This work was carried out at the University of California, Berkeley, during a sabbatical leave of LF from the University of Glasgow, as well as at the University of Glasgow where HSH is grateful for hospitality. We are grateful to K. Shibata and the organizers of the workshop in Fairbanks, Alaska, where this paper was originally presented. LF gratefully acknowledges financial support by the European Commission through the SOLAIRE Network (MTRN-CT-2006-035484) NASA provided support under grant NAG-5-12878 and contract NAS5-38099.

\section{References}

Allred, J. C., S. L. Hawley, W. P. Abbett, and M. Carlsson, Radiative hydrodynamic models of the optical and ultraviolet emission from solar flares, ApJ, 630, 573-586, 2005.

Arber, T. D., M. Haynes, and J. E. Leake, Emergence of a flux tube through a partially ionized solar atmosphere, ApJ, 666, 541, 2007.

Aschwanden, M. J., Particle acceleration and kinematics in solar flares, Space Sci. Rev., 101, 1-227, 2002.
Banks, P., Collision frequencies and energy transfer. ions, Planet. Space Sci., 14, 1105-1122, 1966.

Berlicki, A., Observations and modeling of line asymmetries in chromospheric flares, in The Physics of Chromospheric Plasmas, edited by P. Heinzel, I. Dorotovič, and R. J. Rutten, 387-406, 2007.

Biesecker, D. A., D. C. Myers, B. J. Thompson, D. M. Hammer, and A. Vourlidas, Solar phenomena associated with "EIT waves", ApJ, 569, 1009-1015, 2002.

Brown, J. C., The deduction of energy spectra of non-thermal electrons in flares from the observed dynamic spectra of hard X-ray bursts, Solar Phys., 18, 489-502, 1971.

Carrington, R. C., Description of a singular appearance seen in the Sun on September 1, 1859, Mon. Not. R. Astr. Soc., 20, 13-16, 1859.

Emslie, A. G. and P. A. Sturrock, Temperature minimum heating in solar flares by resistive dissipation of Alfvén waves, Solar Phys., 80, 99-102, 1982.

Fletcher, L. and H. S. Hudson, Flare energy transport by Alfvén waves in the impulsive phase, ApJ, 675, 1645-1655, 2008.

Fletcher, L., I. G. Hannah, H. S. Hudson, and T. A. Metcalf, A TRACE white light and RHESSI hard X-ray study of flare energetics, ApJ, 656, 1187-1196, 2007.

Hale, G. E., The spectrohelioscope and its work, ApJ, 71, 73-101, 1930.

Haerendel, G., Commonalities between ionosphere and chromosphere, Space Sci. Rev., 124, 317-331, 2007.

Hudson, H. S., Thick-target processes and white-light flares, Solar Phys., 24, 414-428, 1972.

Hudson, H. S., Implosions in coronal transients, ApJ, 531, L75-L77, 2000.

Hudson, H., Chromospheric flares, in The Physics of Chromospheric Plasmas, edited by P. Heinzel, I. Dorotovič, and R. J. Rutten, 365-386, 2007.

Hudson, H. S., C. J. Wolfson, and T. R. Metcalf, White-light flares: a TRACE/RHESSI overview, Solar Phys., 234, 79-93, 2006.

Kane, S. R. and R. F. Donnelly, Impulsive hard X-ray and ultraviolet emission during solar flares, ApJ, 164, 151-163, 1971.

Kazeminezhad, F. and M. Goodman, Magnetohydrodynamic simulations of solar chromospheric dynamics using a complete electrical conductivity tensor, ApJ (Suppl.), 166, 613-633, 2006.

Lin, R. P. and H. S. Hudson, 10-100 keV electron acceleration and emission from solar flares, Solar Phys., 17, 412-435, 1971.

Longcope, D. and B. T. Welsch, A model for the emergence of a twisted magnetic flux tube, ApJ, 545, 1089-1100, 2000.

Melrose, D. B., Energy propagation into a flare kernel during a solar flare, ApJ, 387, 403-413, 1992.

Miller, J. A., P. J. Cargill, A. G. Emslie, G. D. Holman, B. R. Dennis, T. N. LaRosa, R. M. Winglee, S. G. Benka, and S. Tsuneta, J. Geophys. Res., 102, 14,631-14,659, 1997.

Obayashi, T., Energy build-up and release mechanisms in solar and auroral flares, Solar Phys., 40, 217-226, 1975.

Régnier, S. and E. R. Priest, Nonlinear force-free models for the solar corona. I. Two active regions with very different structure, Astron. Astrophys., 468, 701-709, 2007.

Schwenn, R., Space weather: The solar perspective, Living Rev. Solar Phys., 3(2), 2007.

Stasiewicz, K. et al., Space Sci. Rev., 92, 423-533, 2001.

Sudol, J. J. and J. W. Harvey, Longitudinal magnetic field changes accompanying solar flares, ApJ, 635, 647-658, 2005.

Thomas, R. J. and R. G. Teske, Solar soft X-rays and solar activity. II: Soft X-ray emission during solar flares, Solar Phys., 16, 431-453, 1971.

Thompson, B. J., J. B. Gurman, W. M. Neupert, J. S. Newmark, J.-P. Delaboudinière, O. C. St. Cyr, S. Stezelberger, K. P. Dere, R. A. Howard, and D. J. Michels, SOHO/EIT observations of the 1997 April 7 coronal transient: Possible evidence of coronal moreton waves, ApJ, 517, L151-L154, 1999.

Uchida, Y., M. D. Altschuler, and G. Newkirk, Jr., Flare-produced coronal MHD-fast-mode wavefronts and Moreton's wave phenomenon, Solar Phys., 28, 495-516, 1973.

Veronig, A. M., M. Karlický, B. Vršnak, M. Temmer, J. Magdalenić, B. R. Dennis, W. Otruba, and W. Pötzi, X-ray sources and magnetic reconnection in the X3.9 flare of 2003 November 3, Astron. Astrophys., 446, 675-690, 2006.

Wang, H., Evolution of vector magnetic fields and the August 271990 X-3 flare, Solar Phys., 140, 85-98, 1993.

H. S. Hudson (e-mail: hhudson@ssl.berkeley.edu) and L. Fletcher 